\title{
On the Nature of the 35-day Cycle in X-ray Binary Her-X-1 = HZ Her
}

\author{
Nikolay Shakura*1,2, Dmitry Kolesnikov ${ }^{1}$, Konstantin Postnov ${ }^{1,2}$, Igor Volkov ${ }^{1,3}$, \\ Ilfan Bikmaev ${ }^{2}$, Tatiana Irsmambetova ${ }^{1}$, Rüdiger Staubert ${ }^{4}$, Joern Wilms ${ }^{5}$, \\ Eldar Irtuganov ${ }^{2}$, Pavel Shurygin ${ }^{2}$, Polina Golysheva ${ }^{1}$, Sergey Shugarov ${ }^{1,6}$, Igor \\ Nikolenko $^{3}$, Eugene Trunkovsky ${ }^{1}$, Gabriele Schönherr ${ }^{8}$, Axel Schwope ${ }^{8}$, Dmitry \\ Klochkov ${ }^{4}$ \\ ${ }^{1}$ Sternberg Astronomical Institute, Moscow State University, \\ 119234, Moscow, Russia \\ ${ }^{2}$ Kazan Federal University, \\ Kazan, Russia \\ ${ }^{3}$ Institute of Astronomy RAS, \\ Moscow, Russia \\ ${ }^{4}$ Institute for Astronomy and Astrophysics, \\ Tubingen, Germany, \\ ${ }^{5}$ Astronomical Institute of the University of Erlangen-Nuremberg, \\ Bamberg, Germany, \\ ${ }^{6}$ Astronomical Institute of the Slovak Academy of Scienses, \\ Tatranska Lomnica, Slovakia \\ ${ }^{7}$ Crimean Astrophysical Observatory, \\ Nauchny, Russia \\ ${ }^{8}$ Leibniz Institute for Astrophysics, \\ Potsdam, Germany \\ E-mail: nikolai.shakura@gmail.com,kolesnikovkda@gmail.com
}

The X-ray binary Her X-1 consists of an accreting neutron star and the optical component HZ Her. The 35-day X-ray variability of this system is known since its discovery in 1972 by the UHURU satellite and is believed to be caused by precession of the warped accretion disk tilted to the orbital plane. We assume that the neutron star undergoes free precession and argue that the optical variability of HZ Her can be explained by forced precession of the accretion disk with a similar period as that of the neutron star. The model parameters include a) the intensity (power) of the stream of matter flowing out of the optical star; b) the X-ray luminosity of the neutron star; c) the optical flux of the accretion disk; d) the X-ray irradiation pattern on the donor star; e) the tilt of the inner and outer edge of the accretion disk. A possible synchronization mechanism based on the coupling between the neutron star free precession and the dynamical action of non-stationary gas streams is discussed.

Accretion Processes in Cosmic Sources - II - APCS2018

3-8 September 2018

Saint Petersburg, Russian Federation

* Speaker. 


\section{Introduction}

$\mathrm{HZ} \mathrm{Her} / \mathrm{Her} \mathrm{X}-1$ is an intermediate mass X-ray binary consisting of a $1.8-2.0 M_{\odot}$ evolved sub-giant star and a $1.0-1.5 M_{\odot}$ neutron star observed as X-ray pulsar [1]. The binary orbital period is 1.7 days, the $\mathrm{X}$-ray pulsar spin period is 1.24 seconds. The optical star fills its Roche lobe and an accretion disk is formed around the neutron star. Due to X-ray irradiation, the optical flux from HZ Her is strongly modulated with the orbital period, being first found by the inspection of archive photo-plates [2]. (Note that before X-ray observations, HZ Her was classified as irregular variable).

The X-ray flux curve of Her X-1 is modulated by a 35 day period. Most of the 35 -day cycles lasts 20.0, 20.5 or 21.0 orbital periods (see, e.g., [3],[4],[5]). The 35-day X-ray cycle consists of a "main-on" of seven orbital periods and a "short-on" of five orbital periods, separated by two "offstates" of four orbital cycles each, during which the X-ray flux switches off almost completely. The $\mathrm{X}$-ray observations are well explained by the precessing accretion disk.

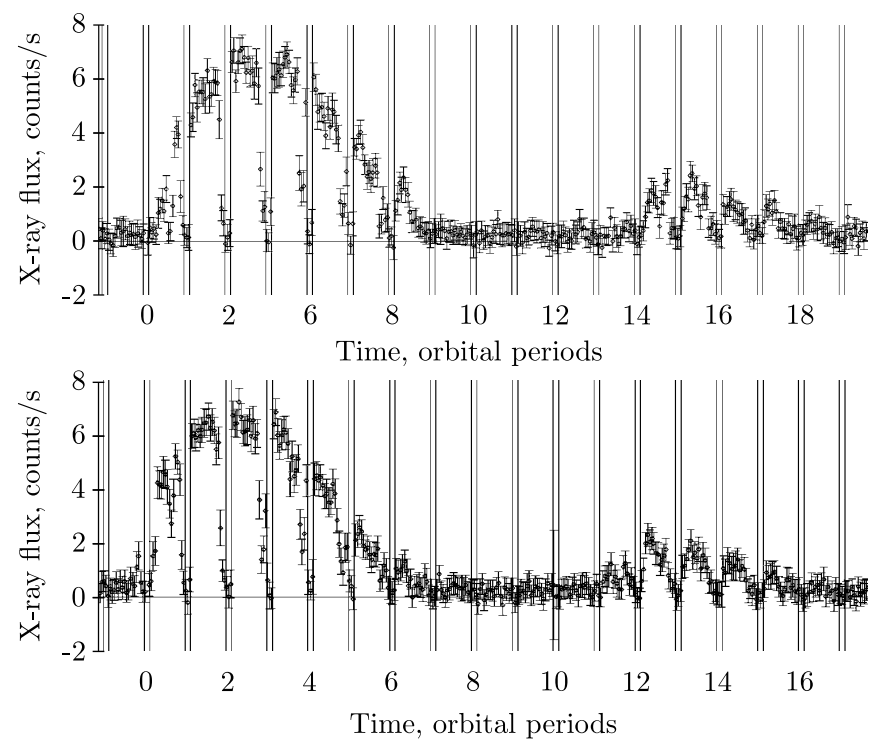

Figure 1: RXTE/ASM light curves of the 35-day X-ray cycle [4]. The vertical lines show eclipses of the X-ray source by the donor star. Top: the "main opening" (also called "turn-on") near orbital phase 0.7. Bottom: the "main opening" near orbital phase 0.2.

\section{35-day cycle}

The 35-day cycle turn-ons most frequently occur at the orbital phases $\sim 0.2$ or $\sim 0.7$, which is due to the tidal nutation of the outer parts of the disk with the double orbital frequency when the angle between the line of sight and the outer parts of the disk changes most rapidly [6], [7], [8]. The 35-day cycle of Her X-1 is explained by the precession of the accretion disk, precessing in the opposite direction of the orbital motion [9], [10]. Soon after the discovery of the X-ray pulsar, NS free precession was suggested to explain the observed 35-day modulation [11]. Later on, EXOSAT observations of the evolution of the X-ray pulse profiles of Her X-1 were also interpreted by free 
precession of the NS [12]. Extensive studies of Her X-1 suggested a warped tilted accretion disk around the NS. Its retrograde precession results in consecutive openings and eclipses of the central $\mathrm{X}$-ray source [8]. The X-ray light curve is asymmetrical between the eclipses due to the scattering of the X-ray radiation in a hot rarefied corona above the disk. Indeed, the X-ray "turn-on" at the beginning of the "main-on" state is accompanied by a significant lack of the soft X-ray flux because of strong absorption. There is no essential spectral change during the X-ray flux increase, suggesting photon scattering on free electrons of the hot corona near the disk inner edge [13], [14], [15], [16]. The X-ray pulse profiles are observed to vary with 35-day phase [12]], [17], [18], [19] changing significantly during the main-on and the short-on. Such changes are difficult to explain using the precessing disk only. The X-ray RXTE/PCA pulse evolution with 35-day phase can be explained [20] by NS free precession with a complex magnetic field structure on the NS surface. In this model, in addition to the canonical field (a magnetic dipole field), arc-like magnetic regions around the magnetic poles are included, which is a consequence of a likely non-dipole magnetic field [21], [22]. Another explanation of the changes of the X-ray flux and X-ray pulses rely on the partial blocking of the NS surface by the inner part of the accretion disk [18],[23]. Such suggestions require fine-tuning of the angle of the inner part of the disk. For reasonable assumptions for the inner radius $R_{i n} \approx 100 R_{N S}$, the accuracy of the angle of the inner disk should be $\theta_{\text {in }} \sim R_{N S} / R_{\text {in }} \approx 0.5^{\circ}$. Our model requires only $\theta_{\text {in }}>0$ at the short-on. Moreover, the hot corona above the inner edge of the disk has a finite opacity and it blurs the X-ray pulses, but in model [23] the inner edge should be sharp in order to partially cover the NS surface.

\section{Modeling of optical light curves of $\mathrm{HZ} \mathrm{Her}$}

Here we performed a modeling of long-term B-light curves. The photometrical light curve was constructed using the following observations: 1972 - 1998 data compiled from [24], [25], [26], [27], [28], [29], [30], [31], [32], [33], [34], [35], [36], [37], [38], [39], [40] ( $\approx 5800$ points); 2010 - 2018 data were obtained by the present authors $(\approx 7600$ points $)$.

The model includes two basic components:

a) an inclined, warped, forced precessing accretion disk;

b) a freely precessing neutron star.

The shape of the model optical light curve strongly depends on the X-ray shadow on the optical star produced by the warped accretion disk and the X-ray irradiation pattern. The shadow is calculated as follows. The disk is split along the radius in a finite number of rings and the solid angle between each $i$-th and $i+1$-th ring is calculated, giving the $i$-th element of the shadow. As the disk is warped, the $i$-th and $i+1$-th rings lie in different planes. The full shadow is produced by all elements.

The geometrical parameters of the disk are given by the tilt to the orbital plane and the phase disk angle (changing smoothly from the outer to the inner disk edges). The disk phase is counted along the orbital motion. It is set to zero at the moment of the maximal opening of the outer disk with respect to the observer. The tilt and phase angles of the $i$-th ring change linearly from the outer edge to the inner edge. The difference between the inner and outer edge is called the twist angle. The twist angle and the difference between the tilt angle of the outer and inner disk edge determine 
the shadow size. If the twist angle is zero and the tilt of the outer and inner edge is the same, the disk shadow is determined only by the width of the outer disk.

To calculate the X-ray irradiation of the optical star we have used the model [20]. In this model despite of usual rotation of the NS with $P_{r o t}=1.24^{s}$ period there is rotation along one of the three main inertia axes with $P_{p r} \approx 35^{d}$ period due to the free precession (Fig. 2). The angle between the

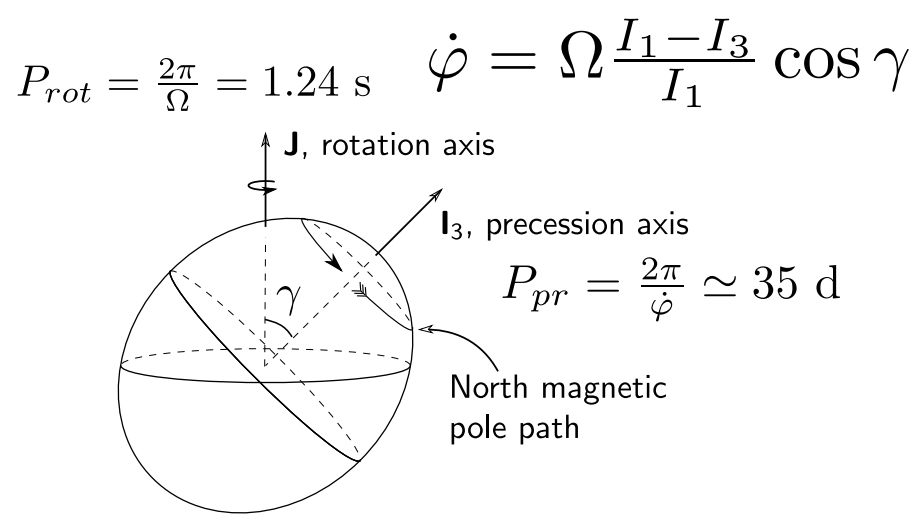

Figure 2: Scheme of the free precession of the NS.

rotation axis and the inertia axes $\gamma=50^{\circ}$. The angle between the magnetic dipole axis and the inertia axis is $30^{\circ}$. During the free precession the magnetic dipole axis covers a small circle on the sky with radius $30^{\circ}$.

At the 35-day phase 0.1 from the "main opening", the angle between the magnetic dipole axis and rotational axis becomes maximal $\left(\beta=50^{\circ}+30^{\circ}=80^{\circ}\right)$. This is the phase zero of the NS free precession. At phase 0.6 , this angle is minimal $\left(\beta=50^{\circ}-30^{\circ}=20^{\circ}\right)$. This is phase 0.5 of the NS free precession.

If the magnetic dipole axis is inclined at $\beta_{0}=\arccos (\sqrt{3} / 3) \approx 54.7^{\circ}$ to the NS rotation axis, the magnetic torque $\mathbf{K}_{\mathbf{m}}$ on the inner edge of the disk vanishes (Fig. 3) [41], [42], [43].

$$
\mathbf{K}_{\mathbf{m}}=\frac{4 \mu^{2}}{3 \pi R_{d}^{3}} \cos \alpha(3 \cos \beta-1)\left[\mathbf{n}_{\omega}, \mathbf{n}_{d}\right]
$$

Here $\mu$ is the magnetic moment of the NS, $R_{d}$ is the inner radius of the disk, $\alpha$ is the angle between NS's rotational axis and the inner disk axis, $\beta$ is the angle between the rotational axis of the NS and the NS's magnetic axis. $\mathbf{n}_{\omega}$ is the unity vector along NS's rotational axis, $\mathbf{n}_{d}$ is the unity vector along the disk's axis.

To the north and to the south of this angle, the magnetic torque is non-zero; the sign of the twist angle is expected to change when crossing this critical angle. Non-zero magnetic torque causes the twist of the inner edge of the disk with respect to the outer edge. In other words, node lines of the inner and outer edges of the disk do not match, there is an angle between them that we call $Z$, which can be both greater or lower than zero depending on the sign of the magnetic torque. The negative sign means that during the precessional motion, the outer disk drags behind the inner disk and vice versa. The value of the angle $\beta$ (the angle between magnetic dipole axis and the rotational axis) becomes equal to $\beta_{0}$ twice during the free precession period. At this moments we should expect 


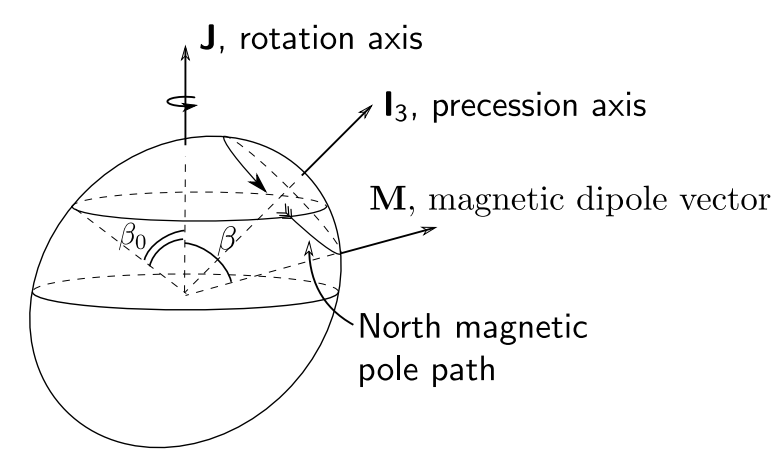

Figure 3: Path of the magnetic dipole due to free precession. Here $\beta$ is the angle between the magnetic dipole axis and the rotational axis. If $\beta=\beta_{0}$, then $K_{m}=0$.

$Z=0$. So, we should expect the opposite sign of $Z$ in the North and in the South. Orientation of the NS rotation axis in the picture plane does not affect the shape of the X-ray pulses, but has a strong effect on the optical light curve. This makes it possible to determine the orientation of the NS spin axis with respect to orbital plane. The angle between the NS spin axis and the projection of the normal to the orbital plane on the sky is called $\kappa$.

The best-fit overall precession phase is obtained for $\kappa=8^{\circ}$ with the 35 -day precession phase 0.1 and 0.6 corresponding to the NS precession phase 0 and 0.5 , respectively.

During the 35-day cycle the disk has a variable angular velocity with a mean value of $18^{\circ}$ per orbit (Fig. 4). The angular velocity of the inclined disk is defined by the tidal forces of the binary system and the dynamic action of the accretion streams. The accretion streams reduce the disk's angular velocity. The disk has the lowest velocity at phases near 0.1 where irradiation by the NS is maximal. Previously, we had suggested a simpler model with a constant angular velocity of the disk and constant $Z$ [44]. The present refined model with a variable angular velocity of the disk provides better fits than the model with constant angular velocity.

When the irradiation is maximal, the accretion flow is also maximal and the dynamic action of the accretion streams on the disk becomes maximal too. On the other hand, at the maximal angular velocity of the disk (at phases near 0.6 where NS's north magnetic pole is most distant from the equator of the NS) the irradiation effect is minimal. We suggest the following formula for the angular velocity of the disk: $\dot{\varphi}\left[{ }^{\circ} / 1.7^{d}\right]=18^{\circ}-A \cos [2 \pi(x-0.05)]$, where $A$ is the semi-amplitude of the angular velocity of the disk, $x$ is the phase of the 35 -day cycle ( $x=0.0$ corresponds to main opening), $\phi$ is the phase angle of the disk. The best estimate for $A$ is $9^{\circ}$.

The tilt of the outer $\theta_{\text {out }}$ and inner $\theta_{\text {in }}$ disk, the X-ray luminosity $L_{x}$ and the contribution to the optical flux from the disk have been optimized at each precession phase (see Table 1). At each precession phase $Z$ takes fixed values from $-100^{\circ}$ to $100^{\circ}$ with $10^{\circ}$ step. We used five disk $h / R$ ratios: $0.05,0.1,0.2,0.3,0.4$; five inclinations: $90,89,88,87,86$; several angular velocity amplitudes $A$. $\theta_{\text {out }}$ and $\theta_{\text {in }}$ as well as accociated parameters shown in the table 1 are satisfying the $\mathrm{X}$-ray visibility in the main-on and the short-on, $\theta_{\text {in }}$ does not exceed $\theta_{\text {out }}$ by more than $2^{\circ}$. Finally, parameters shown in the Table 1 represent the $30 \%$ top best $\chi^{2}$ models.

Table 1 shows the best-fit parameters of the model. Best $Z$ values are shown in Fig. 5. Fig. 6 shows the synthetic light curves with best-fit parameters and observed B light curves of HZ Her. 


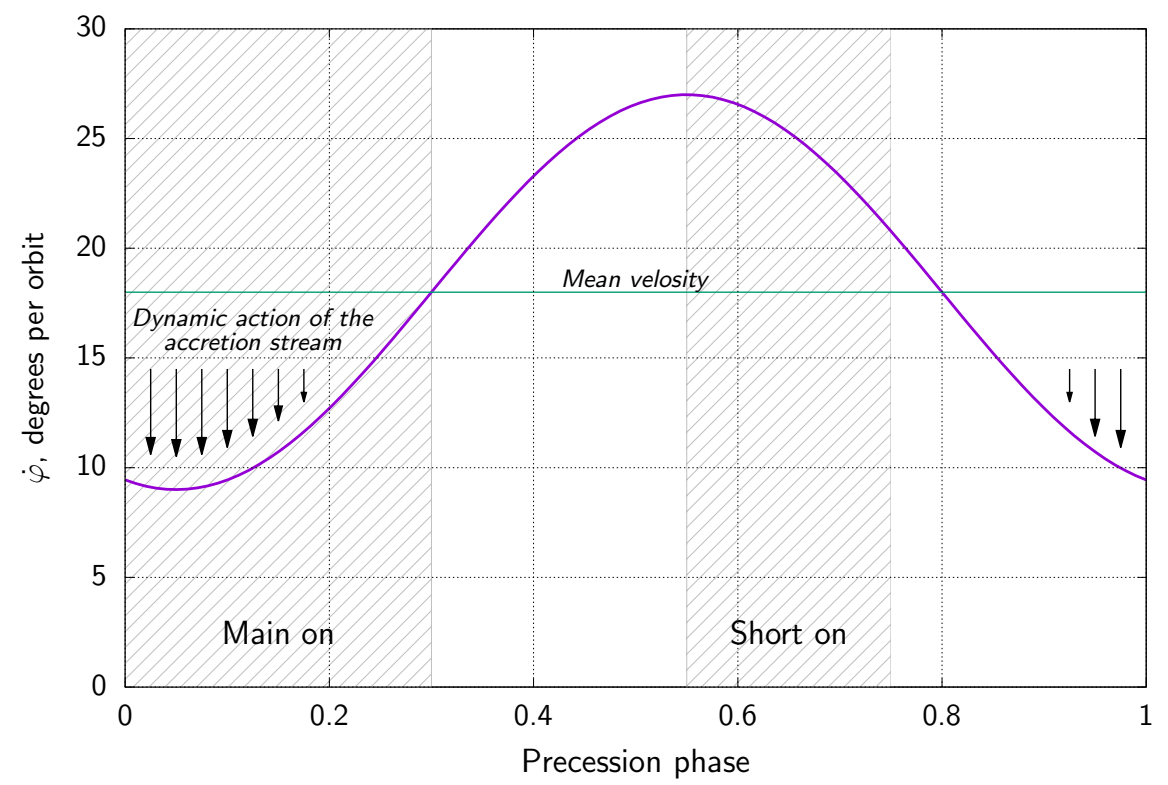

Figure 4: Disk precession variable velosity.

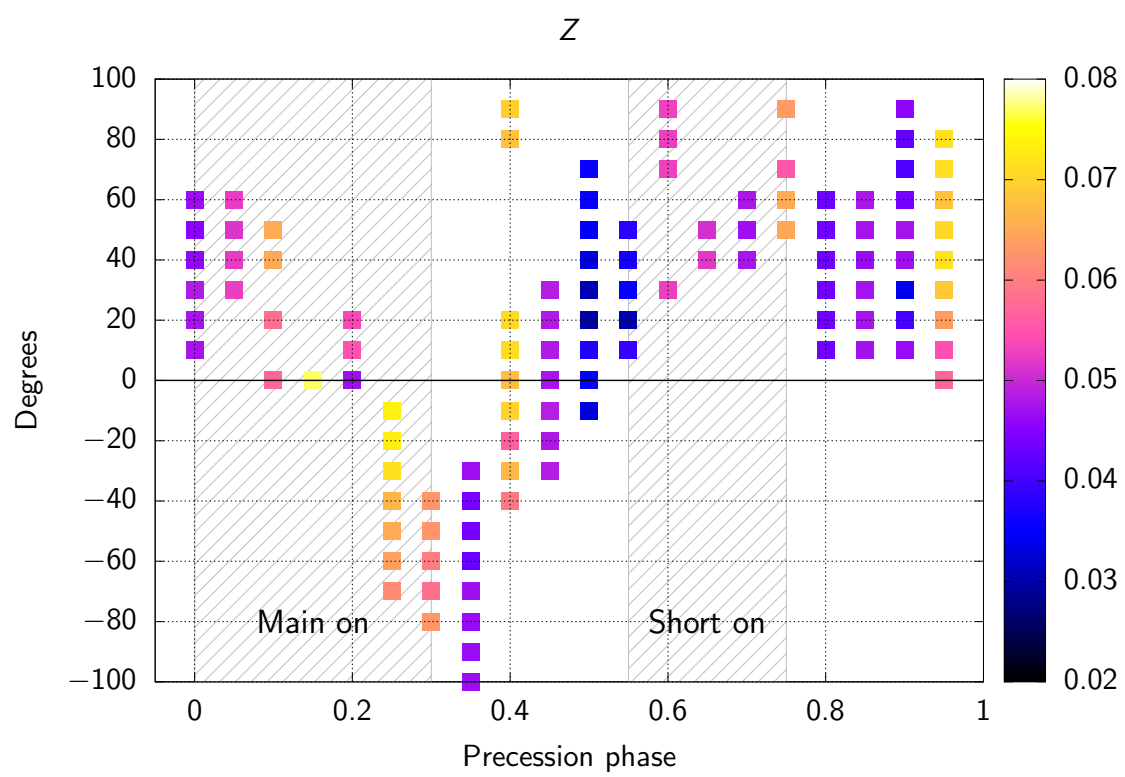

Figure 5: The angle between the inner and outer edges of the disk as a function of the disk precession phase

The modeling was not performed for the optical phase intervals $0.0-0.13$ and $0.87-1.0$. At these phases, the disk is obscured by the optical star. The brightness distribution over the disk is complex, and we leave its study for future work.

Several peak-like features around the first five precession phases should be noted (red dots in Figure 6). These features were observed for the first time by [37], [45], [39] and were investigated 
for the first time by [46]. This is the result of non-stationary streams striking the accretion disk. These streams form an important part of the general nonlinear dynamics of the system. An original modeling of these streams was done by [47]. The streams synchronize the precession of the accretion disk to the precession of the NS. We note that such a situation is realized only if the NS rotation axis is misaligned with the angular orbital momentum (the non-zero angle $\kappa$ in our notation).

The optical light curves demonstrate the secondary minimum near precession phase 0.25 because of the passing of the disk and of the widest part of the shadow above the irradiated part of the optical star at the orbital phase about 0.5 . The secondary minimum is absent at precession phases near 0.75 because the disk is projected onto its own shadow on the optical star surface.

\section{Acknowledgements}

This research has been supported by the RSF grant No. 14-02-00146 (creation of the program and modeling), and the RFBR grant No. 18-502-12025 (carrying out of the observations and data processing), and DFG grant No. 259364563 (processing of the X-ray data), and the grant of the government support of the Kazan (Privolzhsky) Federal University, and support program of the Leading Science School MSU (Physics of Stars, Relativistic Compact Objects and Galaxies). E. Trunkovsky and S. Shugarov express their gratitude to T. Khruzina for help in processing the raw data. Autors express gratitude to N. N. Shakura for correction of the english version of the text. 
Table 1: Best $\chi^{2}$ models. Inclination is $89^{\circ}, \kappa=8^{\circ}, A=9^{\circ}$.

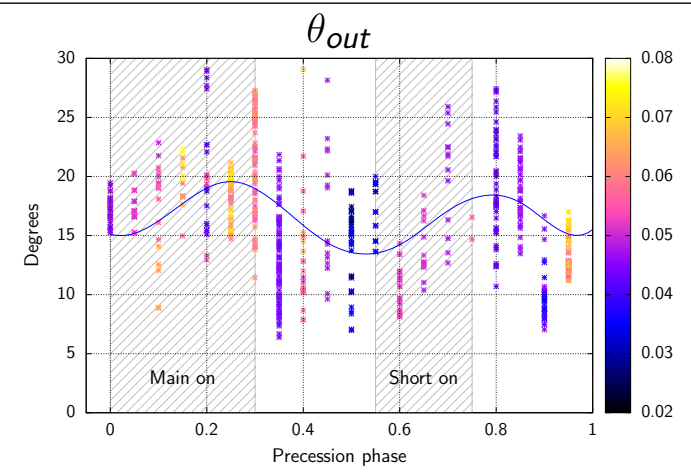

Tilt of the outer $\theta_{\text {out }}$ edge of the disk as a function of the disk precession phase

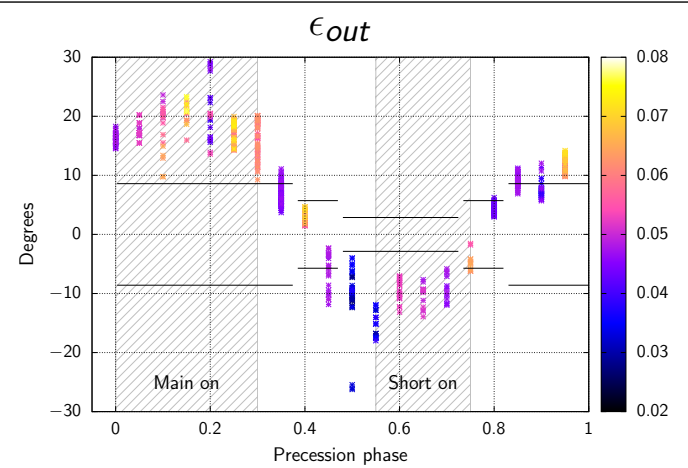

The angle between the direction to the observer and the outer $\varepsilon_{\text {out }}$ disk planes as a function of the disk precession phase. The horizontal lines show the width of the outer disk edge.

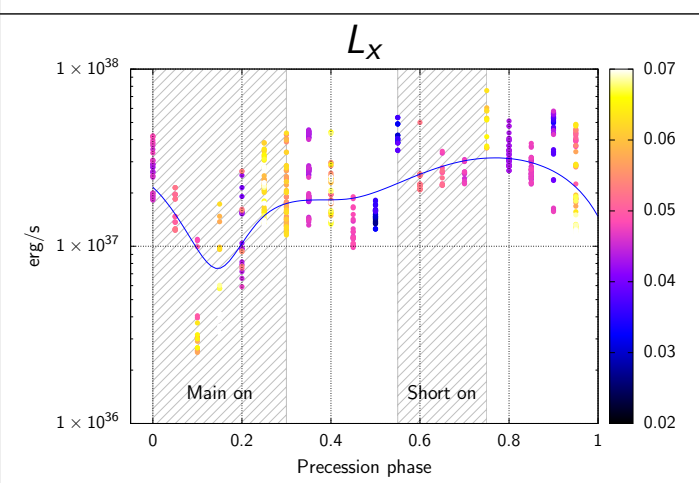

X-ray luminosity as a function of the disk precession phase

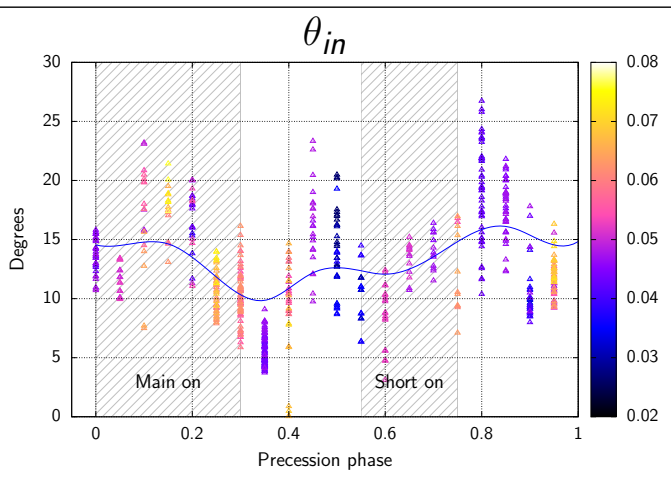

Tilt of the inner $\theta_{\text {in }}$ edge of the disk as a function of the disk precession phase

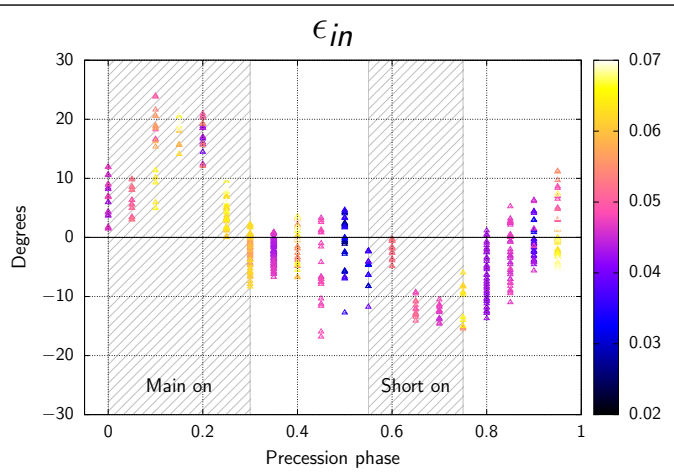

The angle between the direction to the observer and the inner $\varepsilon_{i n}$ disk planes as a function of the disk precession phase

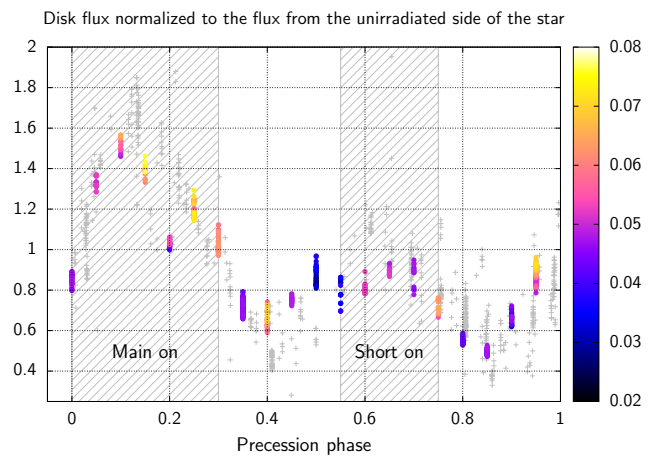

The disk contribution to the optical flux normalized to the flux from the non-irradiated side of the optical star as a function of the disk precession phase 


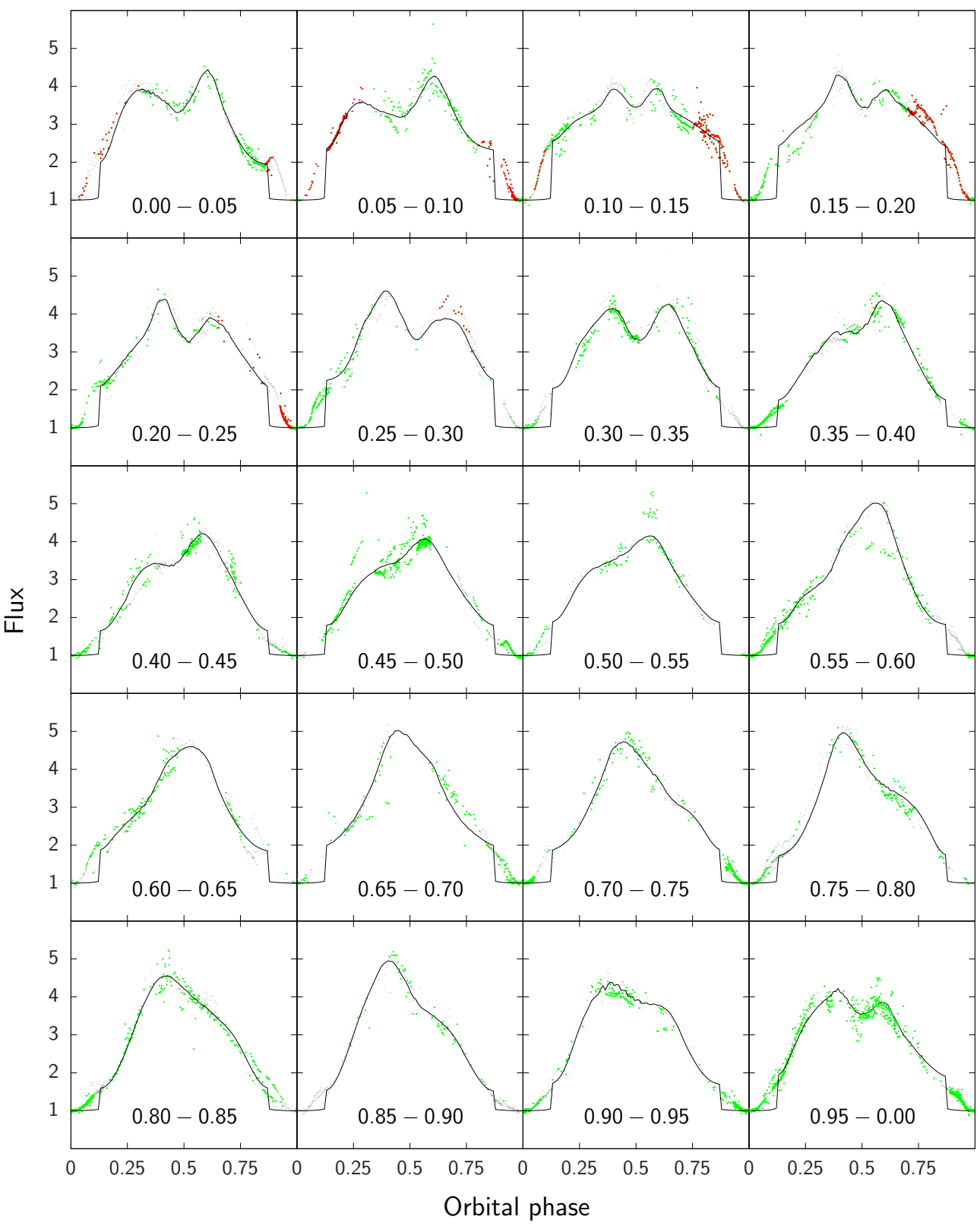

Figure 6: Best-fit synthetic light curves and observed data (points). The 35-day period is divided into 20 phase bins each. The data are colored: in green - the most robust points; in grey — less robust points; in red - points correspondng to the gas stream acting on the disk (bright spot).

\section{References}

[1] H. Tananbaum, H. Gursky, E. M. Kellogg, R. Levinson, E. Schreier and R. Giacconi, Discovery of a Periodic Pulsating Binary X-Ray Source in Hercules from UHURU, ApJ 174 (1972) L143.

[2] A. M. Cherepashchuk, Y. N. Efremov, N. E. Kurochkin, N. I. Shakura and R. A. Sunyaev, On the 
Nature of the Optical Variations of HZ Her = Her X1, Information Bulletin on Variable Stars 720 (1972).

[3] R. Staubert, M. Bezler and E. Kendziorra, Hercules X-1 - A random walk noise model for the 35-day turn-ons, A\&A 117 (1983) 215.

[4] D. K. Klochkov, N. I. Shakura, K. A. Postnov, R. Staubert, J. Wilms and N. A. Ketsaris, Observational manifestations of the change in the tilt of the accretion disk to the orbital plane in her $x$-1/hz her with phase of its 35-day period, Astronomy Letters 32 (2006) 804.

[5] N. I. Shakura, N. A. Ketsaris, M. E. Prokhorov and K. A. Postnov, RXTE highlights of the 34.85-day cycle of HER X-1, MNRAS 300 (1998) 992 [astro-ph/9806126].

[6] J. I. Katz, Thirty-five-day Periodicity in Her X-1, Nature Physical Science 246 (1973) 87.

[7] A. M. Levine and J. G. Jernigan, On the orbital phase dependence of the turn-on times of Hercules X-1, ApJ 262 (1982) 294.

[8] R. Giacconi and R. Ruffini, eds., Physics and astrophysics of neutron stars and black holes, 1978.

[9] D. Gerend and P. E. Boynton, Optical clues to the nature of Hercules X-1/HZ Herculis, ApJ 209 (1976) 562.

[10] N. I. Shakura, N. A. Ketsaris, M. E. Prokhorov and K. A. Postnov, HER X-1: a New Model for X-Ray Dips, Astrophysical Letters and Communications 38 (1999) 165.

[11] K. Brecher, Her X-1: A Processing Binary Pulsar?, Nature 239 (1972) 325.

[12] J. Truemper, P. Kahabka, H. Oegelman, W. Pietsch and W. Voges, EXOSAT observations of the 35 day cycle of Hercules X-1 Evidence for neutron star precession, ApJ 300 (1986) L63.

[13] R. H. Becker, E. A. Boldt, S. S. Holt, S. H. Pravdo, R. E. Rothschild, P. J. Serlemitsos et al., Spectra of Hercules X-1 near a turn-on in the 35 day cycle, ApJ 214 (1977) 879.

[14] P. J. N. Davison and A. C. Fabian, Copernicus and Ariel Vobservations of Hercules X-1, MNRAS 178 (1977) $1 \mathrm{P}$.

[15] A. N. Parmar, P. W. Sanford and A. C. Fabian, Further Ariel V observations of Hercules X-1, MNRAS 192 (1980) 311.

[16] M. Kuster, J. Wilms, R. Staubert, W. A. Heindl, R. E. Rothschild, N. I. Shakura et al., Probing the outer edge of an accretion disk: a Her X-1 turn-on observed with RXTE, A\&A 443 (2005) 753 [astro-ph/0507198].

[17] J. E. Deeter, D. M. Scott, P. E. Boynton, S. Miyamoto, S. Kitamoto, S. Takahama et al., The 35 Day Evolution of the Hercules X-1 Pulse Profile: GINGA Observations and Their Implications, ApJ 502 (1998) 802.

[18] D. M. Scott, D. A. Leahy and R. B. Wilson, The 35 Day Evolution of the Hercules X-1 Pulse Profile: Evidence for a Resolved Inner Disk Occultation of the Neutron Star, ApJ 539 (2000) 392 [astro-ph/0002327].

[19] R. Staubert, D. Klochkov, D. Vasco, K. Postnov, N. Shakura, J. Wilms et al., VizieR Online Data Catalog: Her X-1 pulse profiles (Staubert+, 2013), VizieR Online Data Catalog 355 (2013).

[20] K. Postnov, N. Shakura, R. Staubert, A. Kochetkova, D. Klochkov and J. Wilms, Variable neutron star free precession in Hercules X-1 from evolution of RXTE X-ray pulse profiles with phase of the 35-d cycle, MNRAS 435 (2013) 1147 [1307.6026]. 
[21] N. I. Shakura, K. A. Postnov and M. E. Prokhorov, Presence of a Quadrupole Component of the Magnetic Field Near the Surface of the Neutron Star in HERCULES-X-1, Soviet Astronomy Letters 17 (1991) 339.

[22] I. E. Panchenko and K. A. Postnov, Hercules X-1 pulse profile simulation, A\&A 286 (1994) 497.

[23] E. K. Sheffer, I. F. Kopaeva, M. B. Averintsev, G. S. Bisnovatyi-Kogan, I. M. Golynskaya, L. S. Gurin et al., X-ray studies of the pulsar Hercules X-1 from the Astron space station, Astronomicheskii Zhurnal 69 (1992) 82.

[24] L. Petro and W. A. Hiltner, Light Curve of HZ Herculis, ApJ 181 (1973) L39.

[25] A. Davidsen, J. P. Henry, J. Middleditch and H. E. Smith, Identification of the X-Ray Pulsar in Hercules: a New Optical Pulsar, ApJ 177 (1972) L97.

[26] P. E. Boynton, R. Canterna, L. Crosa, J. Deeter and D. Gerend, Color photometric study of HZ Herculis., ApJ 186 (1973) 617.

[27] V. M. Lyutyj, , Peremennye zvezdy 18 (1973) 41.

[28] S. A. Grandi, P. M. N. O. Hintzen, E. B. Jensen, A. E. Rydgren, J. S. Scott, P. M. Stickney et al., The effects of the 35-day X-ray cycle on the light curve of HZ Herculis., ApJ 190 (1974) 365.

[29] V. M. Luytyi, , Astronomicheskii Zhurnal 50 (1973) 3.

[30] A. M. Cherepashhuk, V. M. Kovalenko, O. N. Kovalenko and A. V. Mironov, Photoelectric ubv-observations of the binary $x$-ray systems hz her (her $x$-1) and v1357 cyg (cyg $x$-1) (in russian), Peremennye zvezdy 19 (1974) 305.

[31] I. B. Voloshina, V. M. Luytyi and E. K. Sheffer, Some features of the photometric behavior of HZ Her/Her X-1 - Twisted-disk effects, Pisma v Astronomicheskii Zhurnal 16 (1990) 625.

[32] V. M. Lyutyj and I. B. Voloshina, The colorimetric study of HZ Her/Her X-1 in the primary minimum The effects of asymmetry, Pisma $v$ Astronomicheskii Zhurnal 15 (1989) 806.

[33] N. N. Kiliachkov and V. S. Shevchenko, U, B, V, R photometry of HZ Herculis during the 1977 X-ray program, Soviet Astronomy Letters 4 (1978) 356.

[34] N. N. Kilyachkov and V. S. Shevchenko, The light curve of HZ Herculis near minimum, and an interpretation, Pisma v Astronomicheskii Zhurnal 6 (1980) 717.

[35] N. N. Kiljachkov and V. S. Shevchenko, The features of the HZ HER light curve near the minimum, Pisma v Astronomicheskii Zhurnal 14 (1988) 438.

[36] N. N. Kiljachkov, , Pisma v Astronomicheskii Zhurnal 20 (1994) 664.

[37] R. Kippenhahn, H. U. Schmidt and H.-C. Thomas, UBV photometry of HZ Herculis - The shape of the primary minimum, $A \& A 90$ (1980) 54.

[38] S. A. Gladyshev, Ph.D. thesis, 1985.

[39] A. V. Mironov, V. G. Moshkalev, E. M. Trunkovskij and A. M. Cherepashchuk, Four-color photometry of HZ HER in 1982-1984. Optical variability of the system in a period of anomalous X-ray activity, Astronomicheskii Zhurnal 63 (1986) 113.

[40] V. P. Goransky and E. A. Karitskaya 1986.

[41] V. M. Lipunov and N. I. Shakura, The nature of binary X-ray pulsars, Pisma v Astronomicheskii Zhurnal 2 (1976) 343. 
[42] V. M. Lipunov, E. S. Semenov and N. I. Shakura, Accretion disk orientation in binary X-ray pulsars, Astronomicheskii Zhurnal 58 (1981) 765.

[43] V. M. Lipunov, Astrofizika nejtronnykh zvezd (Astrophysics of neutron stars). 1987.

[44] N. Shakura, D. Kolesnikov, K. Postnov, I. Volkov, I. Bikmaev, T. Irsmambetova et al., Study of the 35-Day Cycle of the Binary X-ray System of Her X-1 = HZ Her Based on the Broadband Photometric Long-Term Observations, in Modern Star Astronomy. Vol. 1, Astronomy-2018 (XIII Congress of the International Public Organization "Astronomical Society"). Conference Abstracts, Moscow: IZMIRAN, 2018. p. 308-316, vol. 1, pp. 308-316, Sept., 2018, DOI.

[45] H. C. Thomas, J. Africano, A. J. Delgado and H. U. Schmidt, Photometric observations - Is HZ Herculis getting darker?, A\&A 126 (1983) 45.

[46] N. G. Bochkarev and E. A. Karitskaya, Behavior of Matter Near the Outer Region of the Accretion Disk in HERCULES-X-1, in Evolutionary Processes in Interacting Binary Stars, Y. Kondo, R. Sistero and R. S. Polidan, eds., vol. 151 of IAU Symposium, p. 449, 1992.

[47] N. I. Shakura, M. E. Prokhorov, K. A. Postnov and N. A. Ketsaris, On the origin of X-ray dips in HER X-1, A\&A 348 (1999) 917 [astro-ph/9902146]. 